\title{
Multiple Schwannomas of the Spine: Review of the Schwannomatosis or Congenital Neurilemmomatosis: A Case Report
}

\author{
Sang-Hoon Lee, Se-Hoon Kim, Bum-Joon Kim, Dong-Jun Lim \\ Department of Neurosurgery, Ansan Hospital, Korea University College of Medicine, Ansan, Korea
}

Schwannomas are the most common benign nerve sheath tumors originating in Schwann cells. With special conditions like neurofibromatosis type 2 or entity called schwannomatosis, patients develop multiple schwannomas. But in clinical setting, distinguishing schwannomatosis from neurofibromatosis type 2 is challengeable. We describe 58 -year-old male who presented with severe neuropathic pain, from schwannomatosis featuring multiple schwannomas of spine and trunk, and underwent surgical treatment. We demonstrate his radiologic and clinical findings, and discuss about important clinical features of this condition. To confirm schwannomatosis, we performed brain magnetic resonance imaging, and took his familial history. Staged surgery was done for pathological confirmation and relief of the pain. Schwannomatosis and neurofibromatosis type 2 are similar but different disease. There are diagnostic hallmarks of these conditions, including familial history, pathology, and brain imaging. Because of different prognosis, the two diseases must be distinguished, so diagnostic tests that are mentioned above should be performed in caution.

Key Words: Neurofibromatosis type $2 \cdot$ Schwannoma $\cdot$ Schwannomatosis $\cdot$ Spine

\section{INTRODUCTION}

Schwannomas are the most common benign nerve sheath tumors originating in Schwann cells ${ }^{2)}$. Most schwannomas are single sporadic benign neoplasms ${ }^{12)}$. In certain condition such as neurofibromatosis (NF) type 2, patients present multiple schwannomas. Classic hallmark of NF type 2 is bilateral vestibular nerve schwannomas ${ }^{2}$. Some authors described in terms of schwannomatosis, presenting multiple nonvestibular schwannomas with no other signs of NF type $2^{12}$. And recently, this condition was recognized as the third major form of neurofibromatosis. The locus of the disease is placed proximal to NF II locus on chromosome $22 \mathrm{q}^{8,12)}$.

We present a case of multiple schwannomas of the spine related with schwannomatosis, and describe the radiologic and intraoperative findings that clarify the trait of the disease.

\footnotetext{
- Received: April 21, 2015 - Revised: May 20, 2015

- Accepted: June 5, 2015

Corresponding Author: Se-Hoon Kim, MD, PhD

Department of Neurosurgery, Ansan Hospital, Korea University Medical Center,

123 Jeokgeum-ro, Danwon-gu, Ansan, Gyeonggi-do 425-707, Korea

Tel: +82-31-412-5050, Fax: +82-31-412-5054

E-mail: sehoonkim.ns@gmail.com

®This is an Open Access article distributed under the terms of the Creative

Commons Aftribution Non-Commercial License (http://creativecommons.org/ licenses/by-nc/3.0/) which permits unrestricted non-commercial use, distribution, and reproduction in any medium, provided the original work is properly cited.
}

\section{CASE REPORT}

58-year-old male patient visited our institution with progressive intractable both buttock pain that started 10 months ago. $\mathrm{He}$ did not have any relative diagnosed of NF2 in first-degree, and no other family history responded to neurofibromatosis or schwannomatosis. On neurological examination, the patient showed both buttock pain and paresthesia on both lower extremities. Straight leg raising test was negative, and no long tract sign was observed. Sphincter function was normal, and no other specific neurologic deficit was observed including auditory symptoms. In systemic exam, no café-au-lait spot or neurofibroma was seen on trunk. He also complained about palpable mass on his right chest wall.

He had history of surgical excision of palpable masses on his left arm and right thigh in three years ago at other hospital, and schwannoma was confirmed at that time. He also had history of cervical disc herniation at $\mathrm{C6} / 7$, and underwent anterior fusion 2 years ago. Magnetic resonance imaging (MRI) scan was performed for whole spine, and multiple mass lesions were revealed. Total 13 visible masses were seen in the intradural space at $\mathrm{C} 5 / 6$ left, C7 right, T2/3 ventral, T5/6 right, T6/7 left, T10/11 left foramen, L2/3 right foramen, T12/L1 right, L1/2, L2, L3, L4, and L5 (Fig. 1). Preoperative electrophysiology study showed only chronic bilateral L5 radiculopathy. Under the impression of NF type 2 or schwannomatosis, 
we performed brain MRI to rule out NF type 2 . No visible intracranial mass was observed (Fig. 2).

We planned staged operations due to the reasons that his age was 58 , and although his general medical condition was very fair, but operation of long-time could cause unexpected perioperative morbidity. The first operation was performed on thoracolumbar region with intraoperative electrophysiologic monitoring. Through segmental laminectomy, total eight tumors were resected with a permanent pathology of schwannoma. Second operation for cervical and upper thoracic region was followed 3 weeks later. Via en bloc laminoplasty and segmental laminectomy, total four masses were removed. With
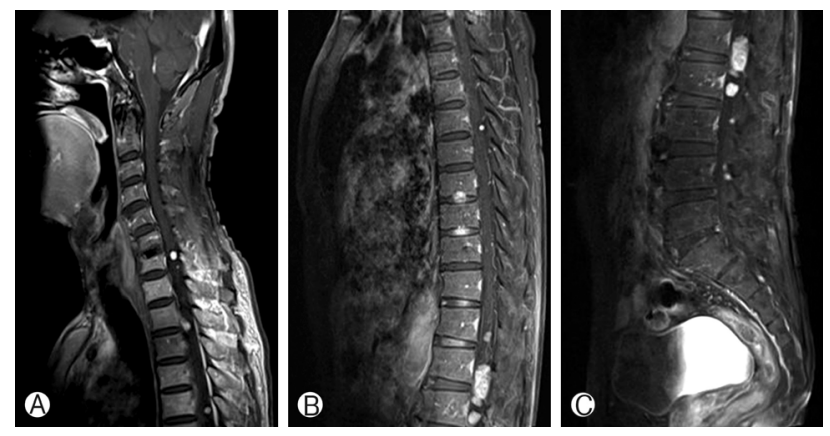

Fig. 1. Total thirteen masses were revealed in Gadolinium-enhanced whole spine $M R$ scan $(A, B$, and $C)$.
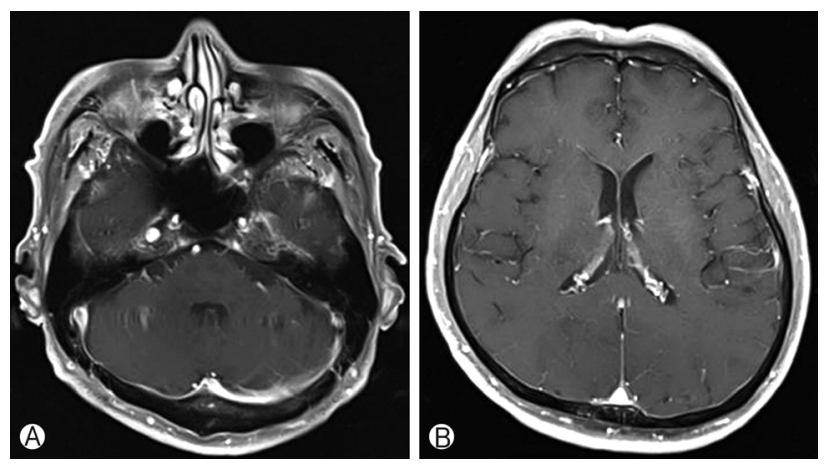

Fig. 2. Brain MRI scan with Gadolinium enhancement revealed no visible intracranial mass ( $A$ and $B)$.
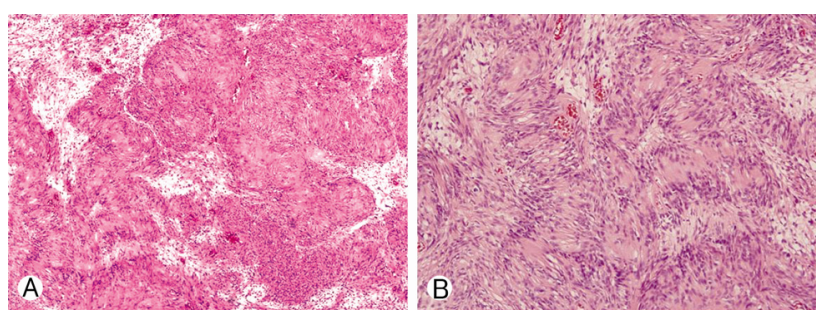

Fig. 3. Pathologic report confirmed diagnosis of schwannoma, $\times 100$, Hematoxilin \& Eosin (H\&E) stain (A). Typical Verocay body is seen $\times 400, H \& E$ stain $(B)$. second operation, palpable mass on the right anterior chest wall was also resected. Pathologic report confirmed schwannoma for all the masses removed (Fig. 3). Postoperatively, the patient showed progressive recovery of both buttock and leg pain, and no neurological or surgical complication was shown. Genetic analysis for NF2 was recommended but refused by the patient due to cost problem.

\section{DISCUSSION}

Schwannomatosis, also known as congenital neurilemmomatosis, is a rare syndrome defined by the development of multiple schwannomas of the peripheral nervous system without concomitant involvement of the vestibular nerves ${ }^{2,6,8,10,12}$. As compared with schwannomatosis, NF type 2 is a well-known genetic disease characterized by the presence of bilateral vestibular schwannomas and multiple cranial and spinal tumors ${ }^{1,3}$. Unlike schwannomatosis, NF type 2 is a serious, progressive, life-threatening disease ${ }^{1)}$. Although the schwannomatosis and NF2 phenotypes are similar, these syndromes are genetically unique and show significant differences in the anatomic distribution of lesions and in clinical presentation, medical management, and patient outcomes. Because of significant differences in disease management and patient mortality, distinguishing these two disease entities is more than an esoteric academic exercise on the part of the diagnostic radiologist ${ }^{6}$. Reported causes of mortality of NF type 2 include swallowing disorders, surgical complication, increased intracranial pressure caused by intracranial tumor, and malignant transformation of preexisting disease ${ }^{1)}$. NF type 1 is a common, autosomal dominant neurocutaneous disorder associated with an increased risk of benign and malignant tumor formation. Disease phenotype is different from NF type 2, that diagnosed with clinical grounds when two or more of the followings are present: (1) a first degree relative with $\mathrm{NF}$ type 1 , (2) six or more café-au-lait spot ( $>0.5 \mathrm{~cm}$ in children and $>1.5 \mathrm{~cm}$ in adult), (3) axillary or groin freckling, (4) two or more Lisch nodules (harmartomas), (5) optic pathway glioma, (6) two or more cutaneous or subcutaneous neurofibromas or one flexiform neurofibroma, (7) a distinct bony dysplasia including sphenoid wing dysplasia or thining of the long-bone cortex with- or without pseudoarthrosis $^{3)}$. As described above, NF type 1 and type 2 present quite different phenotype, but still careful differential diagnosis should be needed. Schwannomatosis represents the third form of neurofibromatosis that differs clinically and genetically from $\mathrm{NF}$ type 1 or 2 , with an annual incidence of 0.58 cases per $1,000,000$ persons. Chronic pain is another typical feature that also presented in our patient. The mean age at diagnosis was 42 years. Our patient's age at presentation was not com- 
patible with the typical mean age but was in the range between 25 and 97 years, which have been reported ${ }^{5,9)}$.

Diagnostic criteria of NF type 2 was presented on Table $1^{11)}$. Our patient showed no auditory symptoms, and no evidence of lower cranial nerve dysfunction. Brain MRI scans showed no visible intracranial lesion, and this was valued that differential diagnosis was made. Diagnostic criteria of schwannomatosis was presented on Table $2^{11)}$. Our patient was 58 years old, and presents multiple schwannoma which was pathologically confirmed. According to the criteria, our patient met definite schwannomatosis.

We performed surgical management without concern of other morbidity of NF type 2 . Generally, the goal of manage-

Table 1. Diagnostic criteria of neurofibromatosis (NF) type $2^{11)}$

A. Bilateral vestibular schwannoma

or

B. NF2 in first-degree relative plus unilateral vestibular schwannoma, or any two of the following neurofibroma, meningioma, glioma, schwannoma, posterior subcapsular lens opacity

or

C. Unilateral vestibular schwannoma plus any two of: neurofibroma, meningioma, glioma, schwannoma, posterior subcapsular lens opacity

or

D. Two or more meningiomas plus unilateral vestibular schwannoma, or any two of: neurofibroma, glioma, schwannoma, or cataract

Table 2. Diagnostic criteria of schwannomatosis ${ }^{11)}$

\footnotetext{
Definite schwannomatosis

A. Age $>30$ years and two or more schwannomas (not intradermal), at least one with histologic confirmation with no evidence of vesitibular tumor on brain magnetic resonance imaging (MRI) scan and no known neurofibromatosis (NF) mutation, or

B. Vesitibular schwannoma (pathologically confirmed) plus first-degree relative who meets the criteria of schwanno matosis

Possible schwannomatosis

A. Age $<30$ years plus two or more schwannomas (not intradermal), at least one with histologic confirmation with no evidence of vesitibular tumor on brain MRI scan and no known NF mutation

B. Age $>45$ years plus two or more schwannomas (not dermal), at least one with histologic confirmation and no symptoms of $8^{\text {th }}$ nerve dysfunction and NF type 2

C. Evidence of a non-vesitibular schwannoma and first-degree relative meeting the criteria for definite schwannomatosis

*All individuals must lack NF type 2 by criteria, lack vestibular schwannoma (by high resolution MRI), lack NF2 in first-degree relative, and lack germline NF type 2 mutations.
}

ment is symptom control, and schwannomatosis-related pain can be treated much like neuropathic pain. As previously known, chronic pain was remained as hallmark of schwannomas, and despite aggressive management with multimodality treatment, most patients did not become pain free (62\% of patient did not become pain free after aggressive medical therapy) ${ }^{7}$. Furthermore, schwannomas occurred to patient with schwannomatosis are become symptomatic lesion earlier than sporadic solitary schwannoma ${ }^{4}$. Our patient was 58-year old, but his life expectancy was not short, so during his life-long follow-up, multiple schwannomatosis could become symptomatic and surgical disease. Because of this aspect, patient with schwannomatosis present depression and anxiety as a common symptom (39\% of women and $17 \%$ of men with schwannomatosis $)^{4}$. And, because of the reason above, our patient showed high anxiety about other non-symptomatic lesions in cervical and thoracic region along with a mass at anterior chest wall, and possibility to progression to symptomatic and surgical disease according to the characteristics of schwannomatosis, we performed staged operations for all visible lesions, though the general rule for asymptomatic lesion is observation ${ }^{6}$. We evaluated the patient's anxiety and concern as the relative indication of surgical treatment.

\section{CONCLUSION}

Patient with severe neuropathic pain from multiple schwannomas, should undergo systemic review and brain imaging for differential diagnosis of NF type 2. Unlike NF type 2, schwannomatosis shows favorable outcomes, and differential diagnosis with appropriate imaging study is absolutely needed.

General treatment rule of asymptomatic schwannoma is observation, but careful surgical treatment could be adopted in a selected patient demonstrating severe anxiety from the remaining tumors. As described above, schwannomas in schwannomatosis occurred early and progress to symptomatic and surgical lesion earlier. So, surgery of schwannomatosis should be concerned as important treatment method. Surgical treatment, however, has the risk of morbidity, and proper treatment decision should be made.

\section{REFERENCES}

1. Aboukais R, Zairi F, Bonne NX, Baroncini M, Schapira S, Vincent $\mathrm{C}$, et al: Causes of mortality in neurofibromatosis type 2. Br J Neurosurg 25:1-4, 2014

2. Chen SL, Liu C, Liu B, Yi CJ, Wang ZX, Rong YB, et al: Schwannomatosis: a new member of neurofibromatosis family. Chin Med J (Engl) 126:2656-2660, 2013 
3. Ferner RE, Gutmann DH: Neurofibromatosis type 1 (NF1): diagnosis and management. Handb Clin Neurol 115:939-955, 2013

4. Gonzalvo A, Fowler A, Cook RJ, Little NS, Wheeler H, McDonald KL, et al: Schwannomatosis, sporadic schwannomatosis, and familial schwannomatosis: a surgical series with long-term follow-up. Clinical article. J Neurosurg 114:756-762, 2011

5. Ioannidis P, Mamouli D, Foroglou N: Expanding schwannomatosis phenotype. J Neurooncol 122:607-609, 2015

6. Koontz NA, Wiens AL, Agarwal A, Hingtgen CM, Emerson RE, Mosier KM: Schwannomatosis: the overlooked neurofibromatosis? AJR Am J Roentgenol 200:W646-653, 2013

7. Merker VL, Esparza S, Smith MJ, Stemmer-Rachamimov A, Plotkin SR: Clinical features of schwannomatosis: a retrospective analysis of 87 patients. Oncologist 17:1317-1322, 2012

8. Murakami Y, Wataya-Kaneda M, Tanaka M, Myoui A, Sakata
Y, Katayama I: Case of schwannomatosis. J Dermatol 36:508511, 2009

9. Plotkin SR, Blakeley JO, Evans DG, Hanemann CO, Hulsebos TJ, Hunter-Schaedle K, et al: Update from the 2011 International Schwannomatosis Workshop: From genetics to diagnostic criteria. Am J Med Genet A 161A:405-416, 2013

10. Qin S, Yang C, Ren H: Cerebral hernia caused by a thoracic surgery for multiple schwannomas in a patient with Neurofibromatosis type 2. Turk Neurosurg 23:245-248, 2013

11. Rodriguez FJ, Stratakis CA, Evans DG: Genetic predisposition to peripheral nerve neoplasia: diagnostic criteria and pathogenesis of neurofibromatoses, Carney complex, and related syndromes. Acta Neuropathol 123:349-367, 2012

12. Seppala MT, Sainio MA, Haltia MJ, Kinnunen JJ, Setala KH, Jaaskelainen JE: Multiple schwannomas: schwannomatosis or neurofibromatosis type 2? J Neurosurg 89:36-41, 1998 